\title{
Schneller durchs Labyrinth der Evidenz
}

\author{
HTA-BERICHTE Ergotherapeuten sehen sich mit vielen Herausforderungen \\ konfrontiert. Evidenzbasiertes Arbeiten gehört sicher zu einer der größten - \\ ernsthaft betrieben kann es sehr anspruchsvoll und zeitaufwendig sein. \\ HTA-Berichte können diese Arbeit erleichtern.
}

E videnzbasierte Praxis sieht vor, dass Ergotherapeuten die beste verfügbare Evidenz nutzen, um ihr therapeutisches Vorgehen zu begründen [1, 2]. Dieser Anspruch klingt plausibel, ist im Arbeitsalltag aber nur schwer zu erfüllen. Er setzt voraus, dass man nach verfügbaren Studien recherchiert, diese bewertet und miteinander vergleicht. Aber mal ehrlich, wann soll eine praktisch tätige Ergotherapeutin das leisten? Glücklicherweise gibt es Quellen, die ihr die Suche nach der besten verfügbaren Evidenz erleichtern. Neben klinischen Leitlinien oder systematischen Übersichtsarbeiten gehören HTABerichte dazu [3].

Evidenzbasierte Praxis leicht gemacht > „Health Technology Assessment“ (HTA) beschreibt ein Verfahren, das medizinische Interventionen und Technologien auf den Prüfstand stellt. Dabei recherchieren Forscher Studien, bewerten diese und fassen ihre Ergebnisse in Berichten zusammen. Die Koordination der HTA-Berichte übernimmt die Deutsche Agentur für HTA (DAHTA), die ihren Sitz in Köln hat und seit 2000 zum Institut für Medizinische Dokumentation und Information (DIMDI) gehört [3, 4].

Ein aktueller HTA-Bericht, an dem die Ergotherapeutin Carola Habermann mitgearbeitet hat, bewertet beispielsweise die Wirksamkeit und Kosteneffektivität der Ergotherapie bei Menschen mit mittleren und schweren Demenzformen (॰ „Demenz“, S. 14). Die Forscher werteten 14 Arbeiten aus und stell- ten einige methodische Mängel fest. Dennoch erscheint ihnen die Ergotherapie empfehlenswert. Demnach profitieren Betroffene unter anderem von einem aktivitätsorientierten Funktions- und Fertigkeitstraining. Außerdem können Ergotherapeuten die Angehörigen durch gezielte Beratungs- und Trainingsangebote unterstützen. Diese wirken entlastend, verbessern die Betreuungskompetenzen und verzögern eine Heimeinweisung [5].

\section{Mit HTA-Berichten ist evidenzbasiertes Arbeiten auch für vielbeschäftigte Ergotherapeuten machbar.}

HTA-Berichte bieten also Therapeuten, Medizinern oder Patienten eine evidenzbasierte Informationsgrundlage. Damit aber nicht genug: Sie können auch gesundheitspolitische Entscheidungen beeinflussen. Zum Beispiel, wenn sie den Gemeinsamen Bundesausschuss dazu veranlassen, Positionen im Leistungskatalog der Gesetzlichen Krankenkasse zu ergänzen, zu erhalten oder zu streichen [6, 7].

Ein weiterer Pluspunkt: Interessierte erhalten die vollständigen Berichte kostenlos über die DAHTA-Datenbank oder über German
Medical Science (๑ „Nützliche Links“). Und das Ganze in deutscher Sprache.

Themenvorschläge erwünscht! > Heißt das jetzt, dass Ergotherapeuten sich ganz auf die DAHTA-Datenbank verlassen können, wenn sie evidenzbasiert arbeiten möchten? So einfach ist es leider nicht. Denn die Anzahl der ergotherapierelevanten HTA-Berichte aus Deutschland ist beschränkt.

Da die Evaluationen sehr zeit- und kostenaufwendig sind, kann die DAHTA pro Jahr nur rund 15 HTA-Artikel veröffentlichen. Das Kuratorium der HTA entscheidet zweimal jährlich darüber, welche Themen Priorität haben. Dieses Kuratorium setzt sich unter anderem aus den Interessenvertretern des Gemeinsamen Bundesausschusses, der Kassenärztlichen Bundesvereinigung, der Krankenversicherungen und Patientenorganisationen zusammen. Ergotherapeuten oder andere Heilmittelerbringer gehören nicht dazu [4, 6, 8]. Wie alle Interessierten können sie ihre Themenvorschläge aber über die Internetpräsenz der DIMDI einreichen (॰ „Nützliche Links“). Nach der Themenauswahl beauftragt die DAHTA qualifizierte Wissenschaftler damit, die HTA-Berichte zu erstellen, und fördert deren Forschung finanziell. Der Entwicklungsprozess unterliegt den „Standard Operating Procedures“ (SOP) und integriert eine mehrstufige Qualitätsprüfung. Dabei sollen HTA-Berichte über die experimentelle Wirksamkeit, die Wirksamkeit unter Alltagsbedingungen und die Kosteneffizienz 
